\title{
A Transparent Loss Recovery Scheme Using Packet Redirection for Wireless Video Transmissions
}

\author{
Chi-Huang Shih, ${ }^{1}$ Ce-Kuen Shieh, ${ }^{1}$ and Wen-Shyang Hwang ${ }^{2}$ \\ ${ }^{1}$ Department of Electrical Engineering, National Cheng Kung University, Tainan 701, Taiwan \\ ${ }^{2}$ Department of Electrical Engineering, National Kaohsiung University of Applied Sciences, Kaohsiung 807, Taiwan
}

Correspondence should be addressed to Chi-Huang Shih, chshih@hpds.ee.ncku.edu.tw

Received 1 October 2007; Revised 14 February 2008; Accepted 17 March 2008

Recommended by F. Babich

\begin{abstract}
With the wide deployment of wireless networks and the rapid integration of various emerging networking technologies nowadays, Internet video applications must be updated on a sufficiently timely basis to support high end-to-end quality of service (QoS) levels over heterogeneous infrastructures. However, updating the legacy applications to provide QoS support is both complex and expensive since the video applications must communicate with underlying architectures when carrying out QoS provisioning, and furthermore, should be both aware of and adaptive to variations in the network conditions. Accordingly, this paper presents a transparent loss recovery scheme to transparently support the robust video transmission on behalf of real-time streaming video applications. The proposed scheme includes the following two modules: (i) a transparent QoS mechanism which enables the QoS setup of video applications without the requirement for any modification of the existing legacy applications through its use of an efficient packet redirection scheme; and (ii) an instant frame-level FEC technique which performs online FEC bandwidth allocation within TCP-friendly rate constraints in a frame-by-frame basis to minimize the additional FEC processing delay. The experimental results show that the proposed scheme achieves nearly the same video quality that can be obtained by the optimal frame-level FEC under varying network conditions while maintaining low end-to-end delay.
\end{abstract}

Copyright (c) 2008 Chi-Huang Shih et al. This is an open access article distributed under the Creative Commons Attribution License, which permits unrestricted use, distribution, and reproduction in any medium, provided the original work is properly cited.

\section{INTRODUCTION}

As different types of wireless networks are converging into the wired Internet,providing end-to-end quality of service (QoS) is essential for video transmission over the wireless Internet. Enabling QoS involves multidisciplinary solutions and the related areas could range from end-users applications to the underlying network architectures. Generally,the QoS support in wireless Internet can be achieved through the network-centric and the end-system centric approaches [1].In the network-centric approach, the integrated service (IntServ) [2] and the differentiated service (DiffServ) [3] are two well-known architectures to support QoS provisioning for the Internet. IntServ and signaling protocols, such as reservation protocol (RSVP), provide the per-flow QoS guarantee. DiffServ provides both the guaranteed and relative QoS by dividing packets into different service classes and forwarding them as different priorities. For wireless networks, there have been many studies related to the QoS provisioning such as the third generation partnership project (3GPP) for UMTS networks [4], IEEE 802.11e for wireless local area networks [5], and IEEE 802.16 for wireless local and metropolitan area networks [6]. The core wireless infrastructures need to provide prioritized QoS services to support various applications with different requirements.

On the other hand, the end-system centric approach is considered as a QoS enhancement solution without the underlying QoS architectures from the network. For the time-varying network conditions, the applications can employ the adaptive control mechanisms to minimize the impairment of video delivery caused by channel errors and congestions during transmission. Congestion control and error control are two main mechanisms to support the robust video transmission. Congestion control mechanism aims at reducing packet loss and delay due to network congestion. Error-control mechanism intends to combat the transmission errors by recovering lost data; for example, two popular error-control schemes are forward error correction (FEC) and automatic repeat request (ARQ). 
To support end-to-end QoS over the wireless Internet, the applications are expected to have two following QoS enhancements: (1) the communication between end users and the underlying QoS architecture for QoS negotiation; and (2) the employment of network adaptive control mechanisms to achieve the effective adaptation of network conditions. The QoS-aware middleware has been introduced to render the application design independent from the underlying network QoS architecture and further achieve the integration of different QoS solutions [7]. It is noted that the major changes to both the end system and legacy video application are necessary, and therefore the deployment of QoS-enabled wireless video services could be slow and difficult. This problem can be solved by improving the degree of transparency to minimize the modifications of the legacy applications. In [8], by adopting so-called QoS library redirection (QLR), applications can set up QoS without source-code modification but the application-dependant library is required for all target applications individually. In [9], a link-layer performance enhancing proxy (PEP) is proposed to cope with the impairment introduced by wireless links over the Internet stack. However, the link-layer approach is inflexible to enable QoS for diverse applications with different requirements.

In this paper, a transparent QoS mechanism (TQM) is proposed to provide a flexible platform to transparently support QoS services. It is noted that this paper focuses on the provision of video services in IP-based wireless networks. Therefore, the proposed TQM transparently supports IP services by employing a packet redirection technique that operates based on the TCP/IP stack. Based on the technique of packet redirection, TQM performs the packet control operations to provide various QoS enhancements for the legacy applications. The proposed TQM aims at facilitating QoS deployment over the wireless Internet. The main features of TQM are: (1) end users can specify which target application receives what types of QoS enhancements and the target application is transparently to be QoS-aware without any modification; (2) no modifications are required to the existing Internet transport protocols; and (3) TQM supports diverse QoS enhancement modules (EM) and thus one can integrate various EMs to maximize the perceived video quality. In this paper, we primarily focus on designing an adaptive error-control scheme for TQM to cope with the varying wireless channel errors, and particularly its packetlevel FEC enhancement module.

FEC introduces the redundancy that trades the additional bandwidth cost to protect video streams from wireless losses. Unfortunately, the effectiveness of FEC decreases since the redundancy cost could lead the self-induced congestion to cause the adverse effects on video quality such as congestion losses due to buffer overflow and the longer end-to-end latency due to queueing delay $[10,11]$. This is significant to the compressed VBR video source since it usually exhibits long-range dependence (LRD) with larger losses and/or delay within a concentrated period [12]. Congestion losses impede the successful loss recovery since the amount of packet losses induced by both wireless error and congestion might exceed the error correction capacity of FEC. The longer end-to-end latency makes packet arrival useless to video decoder with the timing constraint. In addition, the failed redundancy and also the useless video data lead the unnecessary bandwidth waste, studied as the congestion collapse issues by recent works, would degrade the network utility [13]. To enhance the effectiveness of FEC, it is therefore necessary to consider both the loss recovery aspects of FEC and the level of network congestion. Park and Wang [10] consider the optimal problem of designing an adaptive FEC protocol for real-time MPEG video transmission over the Internet without regard to TCP-friendly transmission rate constraints. Their proposed FEC-control method adapts the redundancy degree to perceived packet loss on the network. When increased redundancy results in a nonincreasing recovery performance due to the fact that selfcongestion impedes the timely recovery of video information, the adaptive FEC protocol exponentially decreases the redundancy degree to avoid adverse network effects on video quality. On the other hand, Wu et al. [14] derive the analytical FEC model for a TCP-friendly MPEG video stream with temporal scaling to obtain the optimal reconstruction quality in the presence of packet losses.In their model, FEC is applied to different types of video frames while the temporal scaling technique is used to adjust the stream data rate by discarding frames based on the frame dependency of MPEG video. Yuan et al. [15] present an FEC model, which applies FEC at the group of picture (GOP) level, to increase the error correction capacity of FEC for MPEG video streams within TCPfriendly constraints. Compared with the optimal frame-level FEC technique proposed in [14] by Wu et al., this GOP-level FEC technique requires more computational complexity in average to process a larger amount of video data. However, both techniques rely on the presence of a large buffer to collect an entire GOP for optimally calculating the FEC coding rate in the video sender. This results in a coding buffering delay on the order of GOP duration in the video receiver before the smooth video presentation begins. The coding buffering delay generally contributes to the overall end-to-end delay of video. Usually, the acceptable delay depends on the video applications. For interactive services, such as video conferencing, the end-to-end delay should not exceed 100 milliseconds to ensure good quality [16].

In this paper, the design of FEC-on-TQM integrates several EMs to transparently support robust video transmission on behalf of real-time streaming video applications. FECon-TQM utilizes an instant frame-level FEC technique to minimize the coding buffering delay experienced by the user, while still maintaining near-highest video quality that the optimal frame-level FEC can obtain within the TCPfriendly rate constraints. In order to maintain high video quality with low delay, we first derive a model of video frame priorities based on the temporal dependency of MPEG video to distribute the available TCP-friendly bandwidth budget among video frames. Then the decision of applying FEC to video frames or discarding frames to match the TCP-friendly transmission rate is done on a frame-by-frame basis. To evaluate the performances of the proposed scheme, we constructed the experiments in a controlled network 
environment. The experimental results show that the instant frame-level FEC technique achieves nearly the same video quality that can be obtained by the optimal frame-level FEC while maintaining low end-to-end delay of video. Based on the above mentioned techniques of packet redirection and instant frame-level FEC, FEC-on-TQM carries out the transparent loss recovery without any modification of legacy applications and obtains a high delivered video quality for low-delay video streaming services.

The remainder of this paper is organized as follows. Section 2 describes the basic operating principles of TQM, while Section 3 describes the design of FEC-on-TQM and its implementation issues. Section 4 reviews the instant frame-level FEC control scheme. Section 5 presents and discusses the experimental performance evaluation results. Finally, Section 6 provides some brief concluding remarks and indicates the intended direction of future research.

\section{TRANSPARENT QOS MECHANISM (TQM)}

\subsection{TQM architecture}

The TQM mechanism proposed in this study provides a transparent QoS enhancement for Internet multimedia applications. In order to improve its flexibility, TQM is designed for implementation at the application layer, and therefore enables existing Internet transport protocols to operate in their usual way. As shown in Figure 1, TQM uses two modules, that is, "Flow State" and "QoS Manager", to accommodate the diverse characteristics of existing applications and their underlying transport protocols. When an application is launched, some records are created in the kernel space for system communication and maintenance purposes. Without modifying either the kernel or the application, the virtual flow state module in TQM collects flow information from these records and presents this information to the QoS Manager. The user can then interact with the QoS Manager to specify directly those applications which require enhanced QoS support. Subsequently, the QoS Manager applies the flow information supplied by the flow state module to transparently carry out EM operations on behalf of the applications.

TQM achieves transparent QoS enhancement by means of a packet redirection scheme. Specifically, the implementation of QoS-manager module is based on the functionality of the IP firewall [17] as well as the divert socket [18]. IP firewall filters packets traveling up or down the IP stack; and it defines the target action on these filtered packets, according to firewall rules. Instead of specifying typical target actions such as ACCEPT or DENY, target DIVERT can redirect filtered packets to a divert socket. Divert socket is one element of general BSD socket and can be bound to a specific port of the host for IP packet interception and injection. Since the IP firewall is located at the bottom of the IP stack, it redirects the IP flows which are specified in divert messages received from the QoS manager to a specific system port. The QoS-manager module employs the divert socket to bind this specific port and then receives the IP data packets from it. Using the same port, the EM-

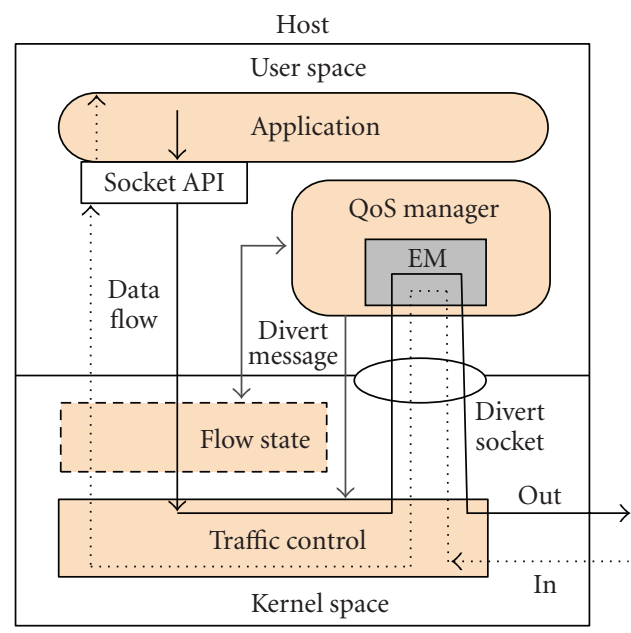

FIgure 1: Basic components and operations of TQM host. Note that sender data flow is marked as "Out" (solid line) and receiver data flow is marked as "In" (dotted line).

processed IP packets are then injected back into the general network protocol stack and are subsequently processed using regular system routines. Accordingly, the packet redirection of TQM operates in a two-way manner. In the TQM sender, the QoS Manager redirects data flows generated by the applications and injects them into the protocol stack for network transmission. Conversely, in the TQM receiver, the QoS Manager redirects the data flows received from the underlying network infrastructure and then injects them into upper-layer applications.

Figure 2 presents a detailed illustration of the major components in the TQM QoS Manager. TQM provides a transparent QoS enhancement capability through the use of data planes and control planes. In the data plane, userspecific flows are identified and the related flow information is passed to the underlying EM. Executing the flow information management function in the data plane involves three separate components, namely the application filter, the user interface, and the application list. Briefly, the application filter collects the flow information relating to launched applications from the flow state module, and users monitor their applications on the application list through the user interface. By accessing the user interface, a user can view those flows which have been launched. Subsequently, he or she can specify the particular flow (or flows) for which enhanced QoS support is required. By querying the application list, the application filter detects and discards any flow information relating to applications which have not been specified by a user.

\section{Application list}

Some applications, for example, DNS query, time services, http services, and so forth, do not have strict QoS requirements. Therefore, TQM uses the application list to indicate the multimedia applications for which the user specifies that QoS enhancement support may be required. The user selects 


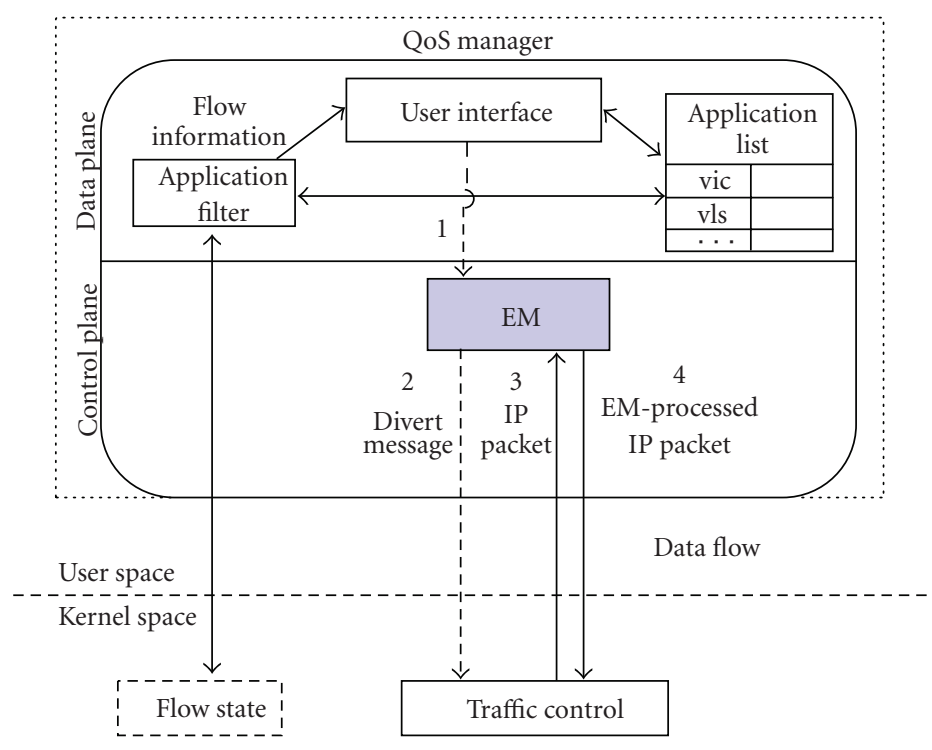

FIGURE 2: TQM architecture.

these specific applications on the application list via the user interface, and can arbitrarily add or delete selection records on demand. By accessing this list, the application filter can indicate to the flow state module the specific applications for which it should collect flow information.

\section{Application filter}

Using the flow state module, the application filter retrieves the flow information required to transparently start QoS sessions on behalf of the applications. Generally, this information is related to five tuples (i.e., the transport protocol, the source IP address, the source port, the destination port, and the destination address). When a user requests support for a specific flow, this information is passed to the control plane, which then establishes the QoS session.

In the control plane, the EMs set up QoS sessions on behalf of the legacy applications. Importantly, the control plane in the proposed TQM mechanism contains many QoS EMs of different types. These EMs may be used either separately or cooperatively in order to carry out various functions. Consequently, TQM provides a flexible and efficient mechanism for the QoS enhancement of diverse applications. Based on the flow information received from the data plane, the EM identifies the user-specified flow and then intercepts the corresponding IP packets to carry out the QoS enhancement process. The EM-processed packets are then returned to the network protocol stack for further processing. Note that IP flow packets may be added, deleted or modified during QoS enhancement depending on the particular EM type.

\subsection{EM types}

TQM comprises a collection of different EMs to support video applications with diverse QoS requirements. Individual EMs may function independently for a specific IP flow or may cooperate with other EMs to improve the overall QoS. The TQM architecture is sufficiently flexible to support both the addition of new EMs and updates of the algorithms upon which the EMs are based. According to the general QoS solutions ranging from the underlying network architectures to upper applications, there are three basic examples of EM to support the QoS requirements of wireless video transmissions, namely, packet mapping control, TCPfriendly congestion control, and adaptive error control. These three EMs are discussed briefly in the following subsections.

\subsubsection{Packet mapping control EM}

In general, QoS architectures employ some form of traffic category concept to support the implementation of scalable and manageable wireless networks with service differentiation capabilities. For example, the 3GPP working group has defined four different QoS classes, that is, conversational, streaming, interactive and background, based on a consideration of the delay sensitivity of different applications. Meanwhile, the 802.11e standard prescribes eight different traffic categories for wireless local area networks. Similarly, the 802.16 standard defines four different types of service flow in wireless metropolitan area networks, namely, unsolicited grant service, real-time polling service, nonreal-time polling service, and best effort service.Through application-specific QoS mapping mechanisms, data flows are assigned to the appropriate traffic category and are then transmitted with the corresponding priority. Furthermore, individual video packets may also be categorized in accordance with their loss and delay properties; and then assigned to different prioritized transmission classes in order to optimize the video quality under given rate or cost constraints.

To accommodate these various strategies, it is necessary to provide differentiation both among multiple flows and 
within single flow. The general differentiation parameters include the IP source/destination address, the protocol, the source/destination port number, and the type of service (TOS) value. In the proposed TQM mechanism, the packet mapping control EM maps the flows identified by the Flow State module to user-specified traffic categories. Meanwhile, differentiation within a single flow is achieved without modifying the applications by transparently diverting the IP packets to the packet mapping control EM. The packet mapping control EM first identifies the packet using appropriate classification criteria (e.g., video frame/layer type) and then maps the packet to the appropriate prioritized class. In mapping the packet to the prioritized class, the EM either directly forwards the identified packets to the underlying network architecture or marks packets with the proper TOS value according to the criteria specified by the underlying architecture before packet forwarding.

\subsubsection{TCP-friendly congestion control EM}

Since the capacity of a wireless channel is scarce and timevarying, bursty losses and excessive delays caused by network congestion can significantly degrade the perceived video quality. Accordingly, congestion control mechanisms aim to minimize the impairment of the delivered video quality caused by network congestion by reducing packet losses and delays. Additionally, video streams must share the available bandwidth equally with other TCP-based flows. Based on the QoS requirements of multimedia transport, TCPfriendly congestion control mechanisms smoothly adjust the transmission rate and avoid increasing the latency by not retransmitting lost packets. Two types of TCP-congestion control mechanism are generally used for multimedia applications, namely sender-based rate adjustment and modelbased rate adjustment. The former mechanism is similar to TCP in that it performs additive increase and multiplicative decrease (AIMD) control at the sender end [19]. Conversely, the latter scheme uses a throughput equation based on a stochastic TCP model to adjust the transmission rate as a function of the loss event rate and the round trip time (RTT) $[20,21]$.

To transport video in a TCP-friendly manner, video applications must match the output rate to the available network bandwidth, as estimated by a TCP-friendly congestion control protocol. One approach for achieving TCPfriendly video transmission is for the video application to use a rate control scheme to regulate the coded bit stream under the constraint of certain given conditions while simultaneously maximizing the user's perception of the media stream quality $[22,23]$. However, this approach generally requires modification of the original legacy applications. An alternative approach is to control the transmission rate of the encoded video stream packets so as to obtain a tradeoff between the degree of TCP-friendliness and the perceived media quality [24]. The TQM mechanism proposed in this study constructs a TCP-friendly congestion control protocol which operates totally transparently to the legacy applications and supports these two approaches to achieve the rate matching. In the first approach, the video transcoding can be used to adapt the output video stream to the available TFRC bandwidth [25]. The general video transcoding parameters consist of frame size, frame rate, and quantization value. In the second approach, the TCP-friendly congestion control EM uses a simple packet control mechanism to either discard packets according to the priority classes they belong to or, if the output rate of the video streams exceeds the TCP-friendly sending rate, to postpone packet transmission until the next transmission period.

\subsubsection{Adaptive error control EM}

Error-control schemes aim at coping with the wireless error problems for high-bit-rate video transmissions over error-prone wireless networks. Typical examples of error control schemes presented in the literature include ARQ and FEC. The objective of both schemes is to obtain a higher data throughput by recovering corrupted packets. However, the two schemes adopt different strategies to achieve this objective. Specifically, ARQ retransmits lost packets, whereas FEC deliberately generates redundant data to enable the reconstruction of any video data which is lost during transmission. Although it has been shown that ARQ is more effective than FEC, its end-to-end retransmissions may impede the timely presentation of video content. Therefore, the FEC scheme is generally preferred for realtime video applications. Since the loss condition changes dynamically in wireless environments, and furthermore, the self-induced congestion caused by the generation of excessive redundant data has an adverse effect on the video quality, it is desirable for FEC-control schemes to have the ability to adapt dynamically to varying network conditions, that is, to changes in the packet loss rate or the level of network congestion. Therefore, the adaptive error control EM in the proposed TQM mechanism installs the FEC encoder in the data sender and the FEC decoder in the data receiver to support the transparent FEC coding of video applications.

The current study focuses primarily on the integration of these three EMs on the proposed TQM to carry out the robust video transmission and the testing of an instant frame-level FEC technique to adjust the number of redundant packets according to the network conditions.

\section{FEC-ON-TQM}

\subsection{Overall structure}

The aim of FEC-on-TQM is to enhance the perceived quality of the delivered video by transparently recovering packet losses on behalf of legacy video applications with no FEC capability. In this study, FEC-on-TQM integrates three EMs mentioned in Section 2.2 to achieve a tradeoff between robust video transmissions and efficient bandwidth utilization. Figure 3 illustrates the end-to-end communication between two TQM hosts. In the video sender, the packet mapping EM identifies the frame type of video packets redirected by the QoS manager and forwards a stream of video frames to the FEC EM. The FEC EM applies 


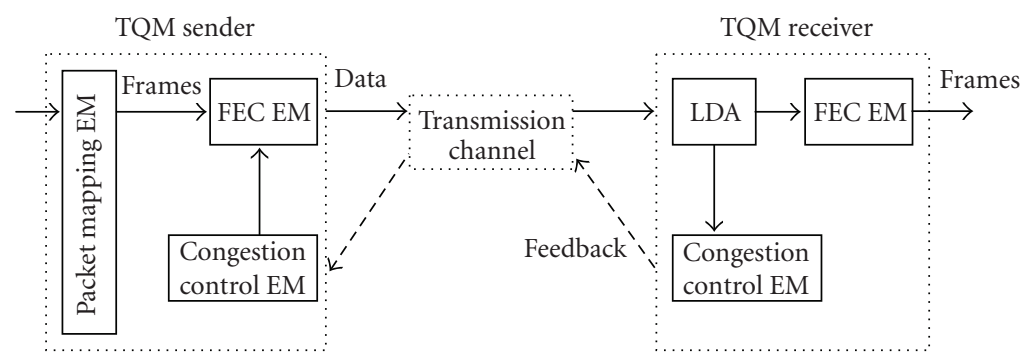

FIGURE 3: FEC-on-TQM transmission scheme.

forward error correction on each frame using the TCPfriendly transmission rate passed on from the congestion control EM, producing a data packet stream including source video packets and redundant packets for network transmission. In the video receiver, the FEC EM collects a sequence of data packets belonging to the same frame and performs a loss recovery operation if required, that is, if a loss condition exists.

To improve the FEC efficiency, the QoS Manager in the video sender requires knowledge of the current network conditions such that the redundancy control function in the FEC mechanism can be adapted accordingly. In FECon-TQM, this is achieved by periodically sending feedback packets from the QoS manager in the video receiver to the QoS manager in the video sender. The feedback packet is useful for the TQM sender in providing packet loss rate and estimating the network round trip time. Since TQM redirects the IP packets of specific applications, packet loss information can be inspected by TQM. In addition to monitoring the packet loss rate, the QoS manager in the video receiver employs a loss differentiation algorithm (LDA) to indicate the congestion signal by discriminating congestion losses from wireless losses for all of the incoming packets, that is, both the source packets and the redundant packets. Two different types of LDA algorithm are commonly employed in receivers nowadays, namely split-connection LDA and end-to-end LDA. The former algorithms, for example, agent-based LDA, differentiate losses using some form of network assistance [26]. Conversely, end-to-end LDA schemes employ a time-based approach and measure queuing delays in an end-to-end manner. The measurement metric used in end-to-end LDA algorithms may be either the packet interarrival time [27] or the relative one-way trip time (ROTT) [28]. Since split-connection LDA algorithms generally require modification of the core network, the current FEC-on-TQM implementation adopts an end-toend LDA scheme. If the LDA regards the loss as a congestion loss, the congestion control EM includes it in the calculation of TCP-friendly transmission rate. Note that in the current deployment, the two end TQM hosts create a new connection to form a feedback channel to carry the packet loss information computed in the receiver through the common socket API. For RTP streams, these feedback messages could be merged into RTCP packets to reduce the control packet overhead [29].

\subsection{FEC-on-TQM design}

In this study, we use systematic Reed-Solomon erasure codes RS $(n, k)$ to protect video data from channel losses. The RS encoder chooses $k$ video data items as an FEC block and generates $(n-k)$ redundant data items for the block. Every data item has its own sequence number used to indicate the corresponding position within the block. With this position information, the RS decoder can locate the position of the lost items and then correct up to $(n-k)$ lost items. Furthermore, the FEC-on-TQM applies a packet-level RS code as FEC since it enables full transparency at the application layer and has a high efficiency over error-prone wireless channels [30]. Packet-level FEC schemes group the source data packets into blocks of a predetermined size $k$, and then encode $n=k+h$ packets for network transmission, where $h \geq 0$ is the number of redundant packets. Provided that $k$ or more packets are successively received, the block can be completely reconstructed. In FEC-on-TQM, one feedback packet is sent from the video receiver to the video sender for every FEC block. Note that packet-level FEC extends the media stream simply by inserting redundant packets into the stream. Therefore, the method requires only minor modification to the source packets and supports a higher degree of transparency.

Figure 4 illustrates the data format of RTP packets through FEC-on-TQM. Other than the IP/UDP/RTP header, FEC is applied only to the source data (i.e., the payload) of the redirected IP packets. Following the FEC coding process, redundant data is generated and grouped into redundant packets in accordance with the header information on the source packets. Furthermore, an additional FEC header is added before the data portion to aid the decoding process. The RTP structures of both the source packets and the redundant packets are defined in accordance with the recommendations of RFC 2733 (In RFC 2733, the length of the RTP header is 12 bytes or more and the length of the FEC header is 12 bytes. In the later IETF Internet draft, the length of the FEC header is 10 bytes [31]) [32]. It is noted that changing the size of the packets or modifying the packet header requires a subsequent adjustment of the length and checksum fields in the IP header for IP validation purposes.Since the additional FEC header may cause the packet length to exceed the path maximum transit unit (MTU) size, the fragmentation may split a long packet into 


\begin{tabular}{|}
\begin{tabular}{|c|c|c|c|c|}
\hline $\begin{array}{c}\text { IP } \\
\text { header }\end{array}$ & $\begin{array}{c}\text { UDP } \\
\text { header }\end{array}$ & $\begin{array}{c}\text { RTP } \\
\text { header }\end{array}$ & Source data \\
\hline $\begin{array}{c}\text { IP } \\
\text { header }\end{array}$ & $\begin{array}{c}\text { UDP } \\
\text { header }\end{array}$ & $\begin{array}{c}\text { RTP } \\
\text { header }\end{array}$ & $\begin{array}{c}\text { FEC } \\
\text { header }\end{array}$ & $\begin{array}{c}\text { Source data } \\
\text { (redundant data) }\end{array}$ \\
\hline
\end{tabular}
\end{tabular}

FIGURE 4: IP datagram format of RTP stream through FEC-onTQM.

two separate packets, resulting in a dependency between the two packets. However, in the receiver end, one of these packets may result in the corruption of the second packet, and hence the efficiency of the FEC recovery process is reduced. Based on the RTP header, FEC-on-TQM identifies the video frame type that the redirected packets belong to by extracting the payload type field and the video-specific header attached to the RTP fixed header. In addition, the timestamp field in the RTP header also helps the frame type extraction since the RTP packets belonging to the same video frame usually tagged with the similar timestamp value.

\section{INSTANT FRAME-LEVEL FEC}

This section develops the instant frame-level FEC technique for real-time streaming video flows within TCP-friendly constraints. To achieve a tradeoff between error correction capacity and low-delay video transmission, the proposed FEC technique determines the amount of FEC required by every video frame in the presence of packet loss when video frames are just dispatched by the streaming video application or the video encoder. A detailed discussion of the proposed instant frame-level FEC is presented in the paragraphs below.

\subsection{TCP-friendly video flows with frame-level FEC}

A TCP-friendly video flow needs to regulate its output rate to match the TCP-friendly transmission rate. Based on a bitrate response function of Reno TCP [33], the TCP-friendly bandwidth $T$ (in bytes/s) is given by

$$
T=\frac{S}{r \sqrt{2 p / 3}+t_{\mathrm{RTO}}(3 \sqrt{3 p / 8}) p\left(1+32 p^{2}\right)},
$$

where $S$ is the packet size in bytes, $r$ is the round-trip time in seconds, $p$ is the current packet loss probability, and $t_{\mathrm{RTO}}$ is the TCP retransmit timeout value in seconds.

In MPEG video, the raw video data are encoded as intracoded $(\mathrm{I})$, predictive $(\mathrm{P})$, and bidirectional $(\mathrm{B})$ video frames. An I frame is encoded without dependence on any past frames. A P frame is encoded based on motion differences from the previous I frame or P frame. B frames are encoded based on the motion differences from the immediate past and future I or P frames. Due to the coding dependency, these three frame types (I, P, and B) have a descending order of importance. After coding, the I, P, and $\mathrm{B}$ frames are arranged in a periodic sequence which is called group of picture (GOP). For instance, a typical GOP pattern is IBBPBBPBBPBB and the GOP size is accordingly 12.
Each frame has to be converted into packets for network transmission and the packet size should not be larger than the path MTU along the traversing links from the sender to the receiver. For a GOP of size $L$, the video sender transmits a series of packet blocks at a frame rate $R_{f}$ frames per second:

$$
k_{1}, k_{2}, \ldots, k_{i}, \ldots, k_{L}
$$

where $k_{i}$ is the number of packets of $i$ th video frame in the GOP. The frame interval is $1 / R_{f}$. Due to the different spatial and temporal activities in the video encoding process, video frames are of variable lengths. According to (1), the available bandwidth allocated to the GOP is given by

$$
T_{\mathrm{GOP}}=T \times \frac{L}{R_{f}} .
$$

In frame-level FEC, the systematic RS erasure code RS $\left(n_{i}, k_{i}\right)$ is applied as FEC to protect video frames. Given the target loss probability $P_{\text {target }}$, the estimated packet loss rate $P$ and fixed $k_{i}$, the lower bound on $n_{i}$ can be computed using

$$
P_{\text {target }}=\sum_{j=n_{i}-k_{i}+1}^{n_{i}}\left(\begin{array}{c}
n_{i} \\
j
\end{array}\right) p^{j}(1-p)^{n_{i}-j} .
$$

According to (4), the FEC encoder generates $h_{i}=n_{i}-k_{i}$ redundant packets for the $i$ th video frame and the number of packets transmitted to the network is $n_{i}$. In the FEC decoder, the $i$ th video frame can be reconstructed when any $k_{i}$ or more packets out of $n_{i}$ transport packets are successfully received. In [34], the picture quality of MPEG-4 is showed to be acceptable at a loss rate of $10^{-5}$ and good at a loss rate of $10^{-6}$. In this paper, the value of $P_{\text {target }}$ in (4) was set to $10^{-6}$ to obtain high-visual-quality video experience.

Figure 5(a) illustrates the processing sequence of source and redundant packets for the instant frame-level FEC. Following the frame $i$ of $k_{i}$ source packets, the desired redundant packets $h_{i}$ are generated and transmitted along with the source packets to combat packet loss in the network. The processing sequences of optimal frame-level FEC and GOPlevel FEC are showed in Figures 5(b) and 5(c), respectively. Both techniques need to defer the FEC processing until an entire GOP arrives. The difference between them is that the optimal frame-level FEC adjusts the amount of FEC per frame while the GOP-level FEC determines an appropriate amount of FEC for a GOP.

For frame $i$, the video data bandwidth $B_{\text {data }}(i)$ and the required FEC bandwidth $B_{\text {req }}(i)$ to achieve the target packet loss probability can be easily computed as follows:

$$
\begin{gathered}
B_{\text {data }}(i)=k_{i} \times S, \\
B_{\text {req }}(i)=n_{i} \times S .
\end{gathered}
$$

Therefore, the total bandwidth that the frame-level FEC can allocate to the GOP must satisfy the following constraint:

$$
\sum_{i=1}^{L} B_{\text {req }}(i) \leq T_{\mathrm{GOP}}
$$




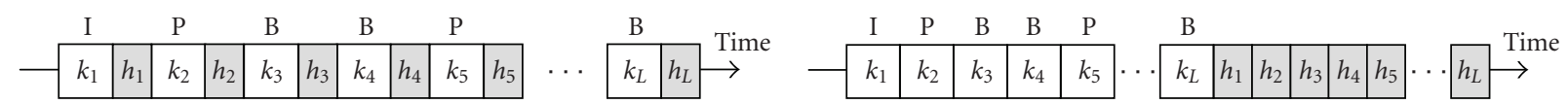

(a) (b)

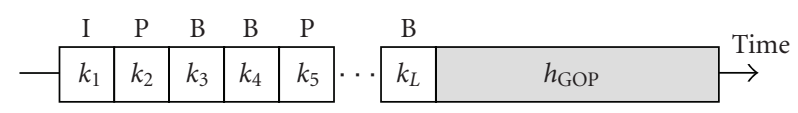

(c)

FIGURE 5: Sequence of source and FEC redundant packets: (a) instant frame-level FEC; (b) optimal frame-level FEC; (c) GOP-level FEC.

To cater for this rate constraint problem, the temporal scaling approach can be used to adjust the amount of video data while preserving the real-time requirement of streaming video applications. In the temporal scaling approach, the video frames with less importance level are discarded before transmission to match the available TCP-friendly transmission rate. For instance, the frame discarding order can be $\mathrm{B}, \mathrm{P}$, and I frame.

\subsection{Classification of video frames}

The GOP pattern in MPEG video is typically arranged as follows:

$$
\begin{aligned}
\mathrm{IB}_{0,0} \cdots \mathrm{B}_{0, N_{\mathrm{BP}}-1} \mathrm{P}_{1} \cdots \mathrm{P}_{m} \mathrm{~B}_{m, 0} \cdots \mathrm{B}_{m, N_{\mathrm{BP}}-1} \mathrm{P}_{m+1} \\
\cdots \mathrm{P}_{N_{\mathrm{P}}} \mathrm{B}_{N_{\mathrm{P}}, 0} \cdots \mathrm{B}_{N_{\mathrm{P}}, N_{\mathrm{BP}}-1}
\end{aligned}
$$

where $N_{\mathrm{P}}$ is the number of $\mathrm{P}$ frames in the GOP and $N_{\mathrm{BP}}$ is the number of $\mathrm{B}$ frames in between an $\mathrm{I}$ and a $\mathrm{P}$ frame or two $\mathrm{P}$ frames. Therefore, the number of $\mathrm{B}$ frames $N_{\mathrm{B}}$ in the GOP is given by $N_{\mathrm{B}}=\left(1+N_{\mathrm{P}}\right) \times N_{\mathrm{BP}}$. Generally, I frame is encoded with high-spatial quality in the GOP and the subsequent $\mathrm{P}$ frames have a gradually degraded spatial quality. Accordingly, the frame type and the frame distance from the reference I frame are two basic criteria to classify video frames in the GOP. Since losing I frame can cause a significant impact on video quality for the entire GOP, I frame has a highest priority. Due to the temporal dependency, $\mathrm{P}$ frames which are closer to the reference I frame has higher priority. As to B frames, which are not used as references of other frames in the GOP, the temporal quality degradation caused by continuous B-frame loss should be considered in classifying video frames. According to the distance of $B$ frames from the reference I frame, $B$ frames are evenly chosen to form $N_{\mathrm{BP}}$ frame groups of size $N_{\mathrm{P}}$ as follows:

$$
\begin{aligned}
& \left\{\mathrm{B}_{0,0} \mathrm{~B}_{1,0} \cdots \mathrm{B}_{N_{\mathrm{P}}, 0}\right\} \\
& \left\{\mathrm{B}_{0,1} \mathrm{~B}_{1,1} \cdots \mathrm{B}_{N_{\mathrm{P}}, 1}\right\} \\
& \cdots \\
& \left\{\mathrm{B}_{0, N_{\mathrm{BP}}-1} \mathrm{~B}_{1, N_{\mathrm{BP}}-1} \cdots \mathrm{B}_{N_{\mathrm{P}}, N_{\mathrm{BP}}-1}\right\} .
\end{aligned}
$$

In each B-frame group, B frames which are closer to the reference I frame have higher priority. Figure 6 shows the

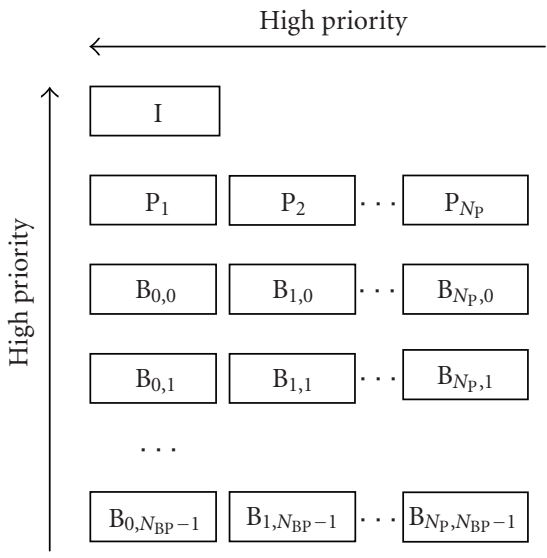

FIgURE 6: Video frame classification within a GOP.

classification of video frames in this study. Therefore, the frame priority sequence in the GOP can be given by

$$
\begin{gathered}
\mathrm{IP}_{1} \mathrm{P}_{2} \cdots \mathrm{P}_{N_{\mathrm{P}}} \mathrm{B}_{0,0} \mathrm{~B}_{1,0} \cdots \mathrm{B}_{N_{\mathrm{P}}, 0} \mathrm{~B}_{0,1} \cdots \mathrm{B}_{N_{\mathrm{P}}, 1} \\
\cdots \mathrm{B}_{0, N_{\mathrm{BP}}-1} \cdots \mathrm{B}_{N_{\mathrm{P}}, N_{\mathrm{BP}}-1} .
\end{gathered}
$$

Based on this prioritized frame sequence, each frame in the GOP pattern is associated with a priority distance from the leading I frame. For instance, with the GOP pattern of ${ }^{\prime} \mathrm{IB}_{00} \mathrm{~B}_{01} \mathrm{P}_{1} \mathrm{~B}_{10} \mathrm{~B}_{11} \mathrm{P}_{2} \mathrm{~B}_{20} \mathrm{~B}_{21} \mathrm{P}_{3} \mathrm{~B}_{30} \mathrm{~B}_{31}$ ", its corresponding priority sequence is " $\mathrm{I}_{1} \mathrm{P}_{1} \mathrm{P}_{2} \mathrm{P}_{3} \mathrm{~B}_{00} \mathrm{~B}_{10} \mathrm{~B}_{20} \mathrm{~B}_{30} \mathrm{~B}_{01} \mathrm{~B}_{11} \mathrm{~B}_{21} \mathrm{~B}_{31}$ " and thus the associated distance sequence of the GOP pattern can be represented as "0 4815926103711 ".

\subsection{Adaptive frame rate allocation}

The proposed FEC technique aims at minimizing the additional FEC processing delay while achieving the near-highest video quality obtained by the optimal frame-level FEC within TCP-friendly rate constraints. To achieve this goal, the FEC performs an online rate allocation for video frames and allows frame discarding to adjust the output data rate without impairing the timing constraints of streaming video. Upon receiving the frame $i$, the required bandwidth $n_{i}$ of the frame is calculated using (4) to achieve the target packet loss probability. Then, the FEC allocates the bandwidth of the frame $i, B_{\text {frame }}(i)$, subject to the transmission rate budget and the frame priorities. After the successful frame bandwidth allocation, the frame $i$ is delivered to the network with its redundancy generated by the FEC. 
Based on the video frame classification described in the previous section, the bandwidth allocated to the video frame should be adaptive to its priority distance from the reference I frame. This adaptive frame rate allocation can be done using a linear weighting factor related to the priority distance of the video frame. Figure 7 illustrates the weighted rate allocation of video frames. Denoting $N$ as the maximum priority distance in the GOP pattern and $d_{i}$ as the priority distance between the frame $i$ and the reference I frame, the corresponding weight $F_{i}$ of the frame $i$ is defined as follows:

$$
F_{i}=\frac{N-d_{i}}{N} .
$$

Using (11) and summing up the weights of all frames, the allocation weight for the GOP can be obtained by

$$
F_{\mathrm{GOP}}=\sum_{i=1}^{N} F_{i}
$$

Since I frame is the first encoded and delivered frame in the GOP, the rate allocation starts by allocating enough FEC bandwidth $\left(n_{1} \times S\right)$, to I frame using (4). After I-frame rate allocation, the available bandwidth for the subsequent frames can be obtained:

$$
T_{\text {avail }}=T_{\mathrm{GOP}}-n_{1} \times S \text {. }
$$

Then, the weighted frame bandwidth of the frame $i$ in the GOP is calculated by

$$
B_{\text {weight }}(i)=\frac{F_{i}}{F_{\mathrm{GOP}}} \times T_{\text {avail }} .
$$

Noted that the frame can be contiguously discarded when the available TCP-friendly transmission rate is low or when fast motion change occurs to induce larger frame size. For the case of contiguous frame discarding, the available bandwidth budget for the next frame accumulates as the number of contiguous discarded frames increases. Let $m$ be the number of frames discarded contiguously before the frame $i$, and let $B_{\text {weight }}(i, m)$ be the weighted frame bandwidth accumulated from the frame $(i-m)$ to the frame $i,(14)$ can be modified as follows:

$$
B_{\text {weight }}(i, m)=\sum_{j=i-m}^{i} \frac{F_{j}}{F_{\mathrm{GOP}}} \times T_{\text {avail. }}
$$

Furthermore, to better utilize the transmission rate budget, the remaining frame bandwidth of the frame $i, B_{\text {rem }}(i)$, is also added to the bandwidth budget of the frame $(i+1)$. Therefore, the available bandwidth for the frame $i$ is given by

$$
B_{\text {avail }}(i)=B_{\text {weight }}(i, m)+B_{\text {rem }}(i-1)
$$

In allocating the frame bandwidth to video frames, we employ the following set of strategies with respect to the type of the frame $i$.

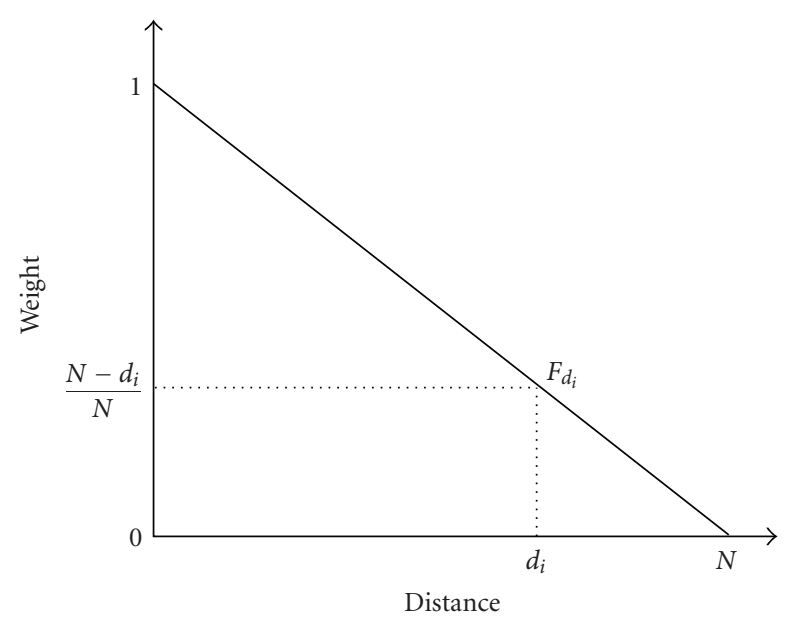

Figure 7: Weighted rate allocation.

(i) For the B frame, if the available bandwidth $B_{\text {avail }}(i)$ is less than the required bandwidth $B_{\text {req }}(i)$, the frame is discarded and accordingly, its available bandwidth will be added to the bandwidth budget of the next frame.

(ii) For the $\mathrm{P}$ frame, if the available bandwidth $B_{\text {avail }}(i)$ is less than the required bandwidth $B_{\text {req }}(i)$, the frame will borrow from the remaining transmission rate budget, $T_{\text {rem }}$, until either the required bandwidth is met or the transmission rate budget is exhausted since the loss of one $\mathrm{P}$ frame can cause the severe quality degradation of other $\mathrm{P}$ and $\mathrm{B}$ frames. The $\mathrm{P}$ frame is discarded when the remaining transmission rate budget is less than the amount of source data $B_{\text {data }}(i)$.

(iii) For the I frame, the I frame is never discarded since the loss of I frame causes the coding failure of an entire GOP.

The adaptive frame rate allocation algorithm used in the instant frame-level FEC is described in Algorithm 1.

\section{PERFORMANCE RESULTS}

\subsection{Experimental setup}

To evaluate the performance of the proposed FEC-on-TQM, a prototype implementation of TQM was developed on the FreeBSD and Linux platforms.As shown in Figure 8, the experimental setup consisted of a video sender, a video receiver and a network bridge, and these three hosts were running on Linux-based x86 PCs. The network bridge bridged packets between networks and produced packet losses and transfer delay for a specified data flow. In the video server, MPEG video sequences were transmitted to the video receiver using the VideoLAN Server (VLS) [35]. FEC-on-TQM EMs were constructed in the video sender and the video receiver, respectively, and an omniscient scheme was built to provide an end-to-end LDA function [36]. The wireless network employed an $802.11 \mathrm{~b}$ access point 


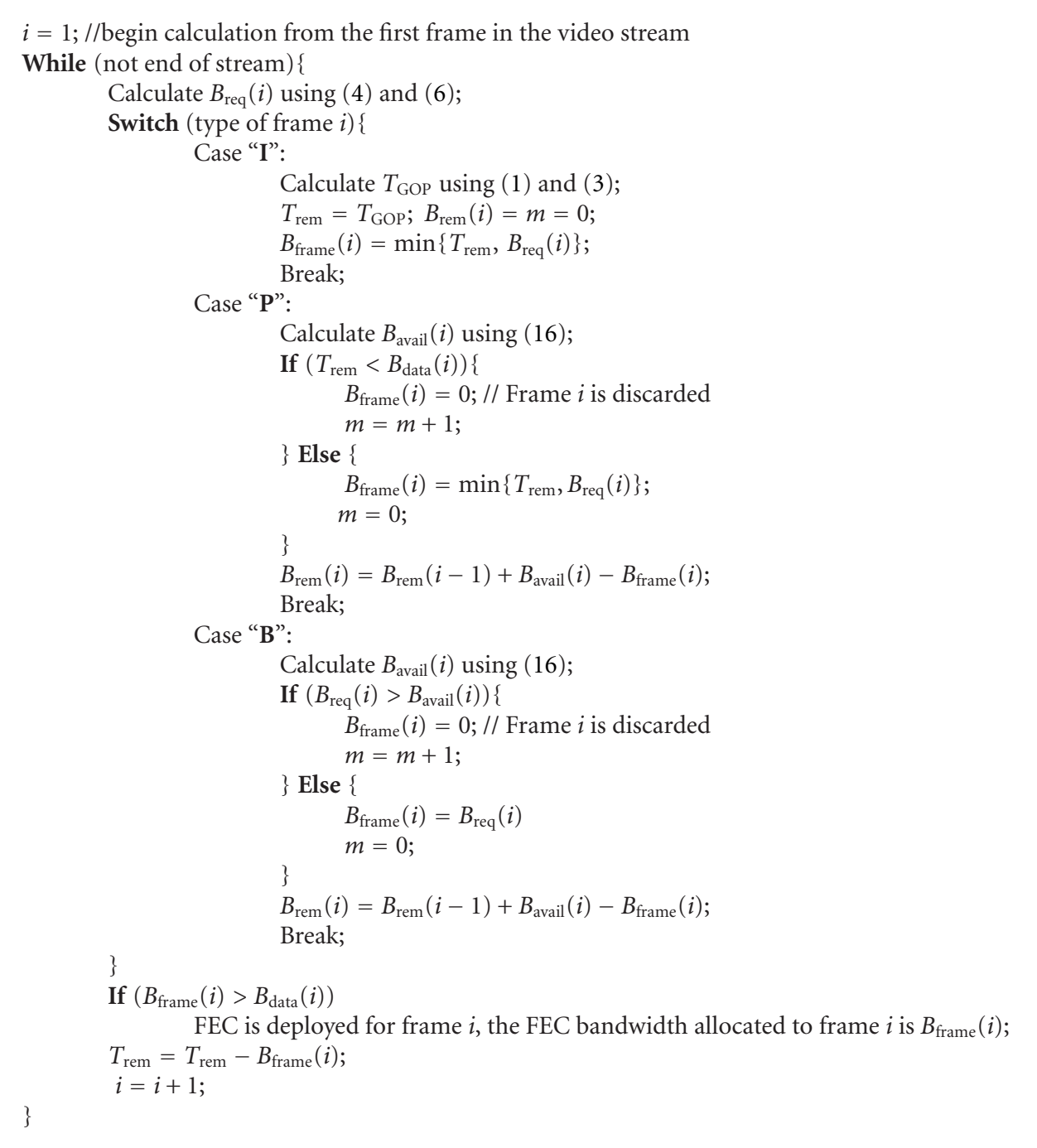

Algorithm 1: Adaptive rate allocation algorithm.

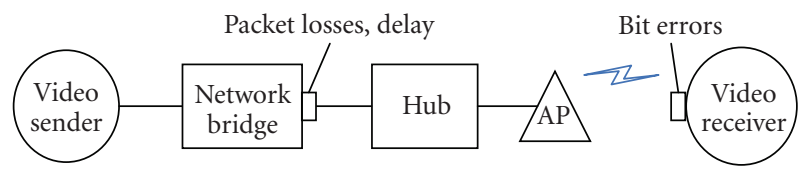

FIgURE 8: Experimental setup.

(AP) operating in distributed coordination function (DCF) mode to connect the video receiver. The video receiver was arranged in clear line of sight (LoS) of the AP to ensure the channel quality and generated packet losses caused by wireless bit errors.

\subsection{TQM overhead}

As described in Section 2, TQM adopts a flow redirection approach to support FEC functionality for legacy applications. In this approach, packets are copied from the kernel space to the user space and are then sent back to the kernel space following processing. Clearly, this processing overhead introduces the additional delays at the sender and the receiver end. In multimedia applications, delay is a major factor influencing the perceived media quality. To evaluate the overhead incurred by the TQM scheme, an experiment was performed to determine the average delay time incurred by the TQM flow redirection process for packets of various sizes. In the experiment, the video sender sent ICMP ping packets to the video receiver, and then waited for the echo packets to be returned. Each echo packet was redirected to the TQM module, processed, and then injected to protocol stack for transmission. The RTT was then measured at the video sender end in order to calculate the difference between the TQM-redirected RTT and the non-TQM-redirected RTT. The corresponding results are presented in Table 1 for ICMP packets of four different sizes. It can be seen that the difference between the directed and nondirected RTTs increases with an increasing packet size. 
However, the difference between the two times is very small. For a 1024-byte ICMP packet, which is around the typical packet size in most video applications, the RTT increases by only $0.276 \%$ when the flow is redirected to TQM. In other words, compared to the total transmission and processing delays incurred along the path between the sender and the receiver, the TQM overhead is relatively minor.

A second experiment was then performed to investigate whether the additional delays caused by TQM and FEC-onTQM would accumulate to such an extent that they affected the overall playout time of video applications. For FECon-TQM, the frame type extraction time, the FEC encoding/decoding time, and the redirection delay are considered to measure the cumulative delay. Using the same setup as that shown in Figure 8, long-lived video sequences were sent from the video sender to the video receiver. The number of FEC redundant packets was set to a static value of 1 for each video frame. Since FEC decoding is not triggered in the TQM receiver under loss-free conditions, an assumption was made that one transported packet per FEC block was lost during transmission. The total playout time was estimated at the receiver and was then compared with the playout time with no flow redirection or cumulative delays. Table 2 presents the results obtained for eight different video sequences. It is observed that the TQM redirection delay does not exceed 90 milliseconds for any of the sequences. Furthermore, for the case of FEC-on-TQM, it can be seen that the FEC coding operations result in an additional cumulative delay of approximately 3-4 milliseconds. In practice, however, this additional delay can be easily absorbed by the application buffer and will have no effect on the user's perception of the delivered video quality. In the current experimental setup, both the video sender and the video receiver are fitted with Pentium-4 $1.8 \mathrm{G}$ processors and $256 \mathrm{MB}$ of memory. The experimental results suggest that this configuration is sufficient to support the proposed TQM.

\subsection{The FEC-on-TQM performance}

To facilitate a controlled environment for performance measurement purpose, the video application at the sender is replaced by an emulator that feeds the frame of stored MPEG-4 video clips at the real-time frame rate to the video receiver. The rest of experimental set-up keeps unchanged. The video sequence "Foreman" of CIF format is used in this experiment. The videoclip is transmitted at a frame rate 25 frames per second and the GOP size is 9. In general, video frames are segmented into packets for transmission. Therefore, the application-level quality depends not only on the successful recovery of lost packets, but also on the dependency relations and delay constraint between the individual video frames. A frame is considered to be decodable at the video receiver when at least a fixed portion DT (decodable threshold) of the data in each frame is received in time and all of the frames it depends on are also decodable. In the experiment, the stream is transmitted in packets of 1 Kbytes and the value of $D T$ is set to 1 for all video frames to observe error performance by dismissing the effect of error resilience features of video transport. The

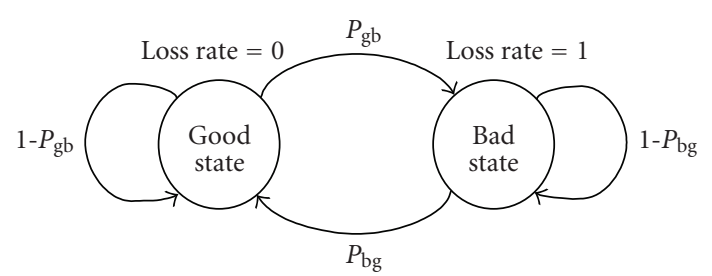

Figure 9: Gilbert model.

video receiver processes the packet arrival in accordance with the hard real-time constraint model presented in [10]. Also, we implement three FEC schemes including the instant frame-level FEC, the optimal frame-level FEC and the GOPlevel FEC scheme on FEC-on-TQM. For all three schemes, TCP-friendly transmission rate is calculated for every GOP. As in [20], $t_{\mathrm{RTO}}$ is typically set to four times of the round trip time $r$. In order to match the TCP-friendly sending rate with real-time constraints, both the optimal frame-level scheme and the GOP-level scheme employ the temporal scaling technique to discard frames based on the video frame priority described in Section 4.2.

In this evaluation, either congestion loss or wireless loss causes the end-to-end packet loss. A Gilbert model or a twostate Markov model is used to generate burst loss patterns over the transmission channel (Figure 9). The two states of the model are Good-state and Bad-state. In the network bridge, we employ a packet-level Gilbert model to drop packets with the parameters of average packet loss rate $\left(P_{B}\right)$ and average burst packet loss length $\left(L_{B}\right)$. In Good-state, a packet is dropped with the probability of 0 , while in Badstate, a packet is dropped with the probability of 1 . The transition probabilities of $P_{\mathrm{gb}}$ and $P_{\mathrm{bg}}$ can be derived by the values of $P_{B}$ and $L_{B}$ as follows:

$$
P_{\mathrm{bg}}=\frac{1}{L_{B}}, \quad P_{\mathrm{gb}}=P_{\mathrm{bg}} \times \frac{P_{B}}{1-P_{B}} .
$$

In the video receiver, on the other hand, a bit-level Gilbert model is used to model packet loss due to bit errors in the wireless channel. This bit-level model is described by an average bit error rate $\left(P_{b}\right)$ and an average burst bit error length $\left(L_{b}\right)$. A packet of size $\tau$ in bits is dropped when at least one bit within $\tau$ bits is lost. Table 3 presents the system parameter settings for the network.

To verify the performance of FEC schemes designed for delay-sensitive video service, we compare the end-to-end frame delay among three FEC schemes built on TQM in different network conditions. The end-to-end frame delay is defined as the time difference between the frame release time in the video sender application and the arrival time of the similar frame in the video receiver application. When the frames are discarded by FEC schemes or are lost during transmission, they are excluded from the calculation of endto-end delay. Figure 10 shows the end-to-end frame delay analysis of three FEC schemes with $P_{B}=0.01$ and $P_{b}=10^{-6}$. It is noted that the instant frame-level scheme performs the FEC encoding process on a frame-by-frame basis, while both the optimal frame-level and the GOP-level scheme 
TABLE 1: Flow redirection overhead.

\begin{tabular}{lcccc}
\hline $\begin{array}{l}\text { ICMP packet } \\
\text { size (bytes) }\end{array}$ & $\begin{array}{c}\text { Non-TQM- } \\
\text { redirected RTT } \\
(\mathrm{ms})\end{array}$ & $\begin{array}{c}\text { TQM-redirected } \\
\text { RTT (ms) }\end{array}$ & $\begin{array}{c}\text { RTT difference/ } \\
\text { RTT difference } \\
\text { RTT }\end{array}$ & $\begin{array}{c}\text { non-TQM-redirected } \\
\text { R6 }\end{array}$ \\
\hline 0.975 & 0.981 & 0.006 & $0.615 \%$ \\
12 & 2.579 & 2.587 & 0.008 & $0.310 \%$ \\
2048 & 4.344 & 4.356 & 0.012 & $0.276 \%$ \\
\hline
\end{tabular}

TABLE 2: Cumulative delay of FEC-on-TQM scheme.

\begin{tabular}{lccc}
\hline Video sequence & Total length & Redirection delay $(\mathrm{ms})$ & Redirection + FEC coding delay $(\mathrm{ms})$ \\
\hline Cars & $56 \mathrm{~min}$ & 86.39 & 89.68 \\
X-Men & $55 \mathrm{~min}, 25 \mathrm{~s}$ & 89.25 & 92.16 \\
Mission impossible & $63 \mathrm{~min}, 6 \mathrm{~s}$ & 85.55 & 88.85 \\
Brokeback mountain & $65 \mathrm{~min}, 39 \mathrm{~s}$ & 83.66 & 86.63 \\
Final destination & $45 \mathrm{~min}, 50 \mathrm{~s}$ & 88.21 & 91.85 \\
The Da Vinci code & $76 \mathrm{~min}, 46 \mathrm{~s}$ & 85.36 & 88.06 \\
Monster house & $45 \mathrm{~min}, 6 \mathrm{~s}$ & 87.85 & 90.98 \\
World Trade Center & $53 \mathrm{~min}, 14 \mathrm{~s}$ & 86.68 & 90.16 \\
\hline
\end{tabular}

TABLE 3: Network settings.

\begin{tabular}{lcc}
\hline Parameters & Scenario 1 & Scenario 2 \\
\hline Delay & $12.5 \mathrm{~ms}$ & $12.5 \mathrm{~ms}$ \\
$P_{B}$ & 0.01 & $0.01,0.02, \ldots, 0.1$ \\
$L_{B}$ & 3 & 3 \\
$P_{b}$ & $10^{-6}, 10^{-5}, \ldots, 10^{-1}$ & $10^{-3}$ \\
$L_{b}$ & 80 & 80 \\
\hline
\end{tabular}

defers the encoding process until all video frames within the GOP are received. From Figure 10, we can observe that: (1) the instant frame-level scheme has much smaller endto-end frame delay than other two schemes and the delay values are below 40 milliseconds (i.e., frame interval); (2) for the optimal frame-level or the GOP-level scheme, the GOP buffering results in a sawtooth delay curve and peaks in the curve are correlated with the periodic insertion of I frames; and (3) the end-to-end frame delays of the GOP-level scheme are slightly smaller than that of the optimal framelevel scheme, since the GOP-level scheme performs the FEC encoding on a GOP and this requires less redundancies for network transmission to protect the entire GOP against the fixed network packet loss.

In the first experimental scenario, the bit error rate is varied from $10^{-6}$ to $10^{-1}$ as the packet loss rate is fixed to 0.01 . Figures 11 and 12 show the average end-to-end frame delay and the average PSNR, respectively. It is noted that for the bit error rate $=10^{-1}$, all packets are lost and none of video frames is available to measure the end-to-end frame delay. From Figures 11 and 12, it can be seen that for the instant frame-level scheme: (1) the average end-toend frame delay is below 40 milliseconds as the bit error rate varies, and (2) the average PSNR curve is close to that of the optimal frame-level scheme and the largest PSNR

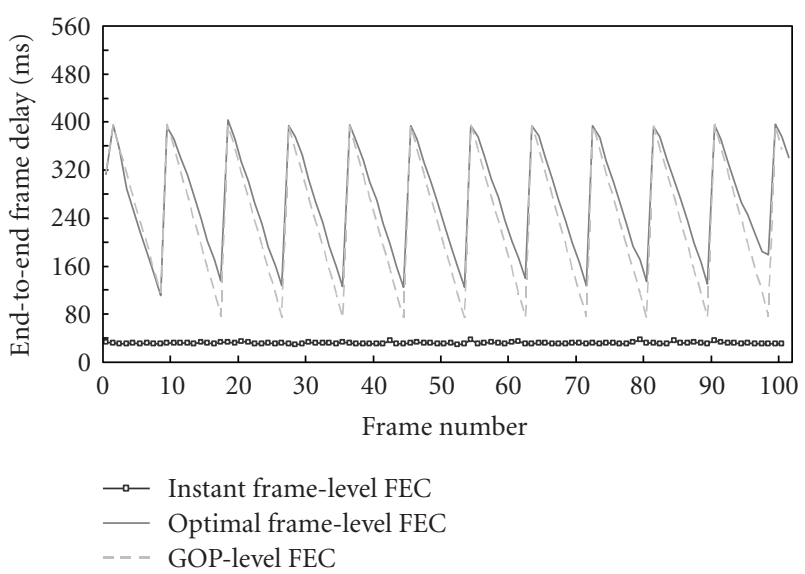

Figure 10: End-to-end timing between frames 1-100 with parameters $P_{B}=0.01, P_{b}=10^{-6}$.

difference of $0.52 \mathrm{~dB}$ occurs as the bit error rate is $10^{-2}$. We also observe that compared to the GOP-level scheme, the optimal frame-level scheme has lower average PSNR value with the larger end-to-end frame delay. Figures 13 and 14 show the performance results for another scenario. As the packet loss rate is increased, the TCP-friendly sending rate decreases accordingly. Besides the similar observations as in the first scenario, the difference in the average PSNR value between the instant frame-level scheme and the optimal frame-level scheme is ranged from $0.01 \mathrm{~dB}$ to $0.71 \mathrm{~dB}$. In Figure 13, the delay gap between the optimal frame-level scheme and the GOP-level scheme becomes large as the packet loss rate exceeds 0.01 . This is because that the amount of discarded frames increases in the optimal frame-level scheme to match the low TCP-friendly sending rate and the frames with high priority, such as I frames, usually 


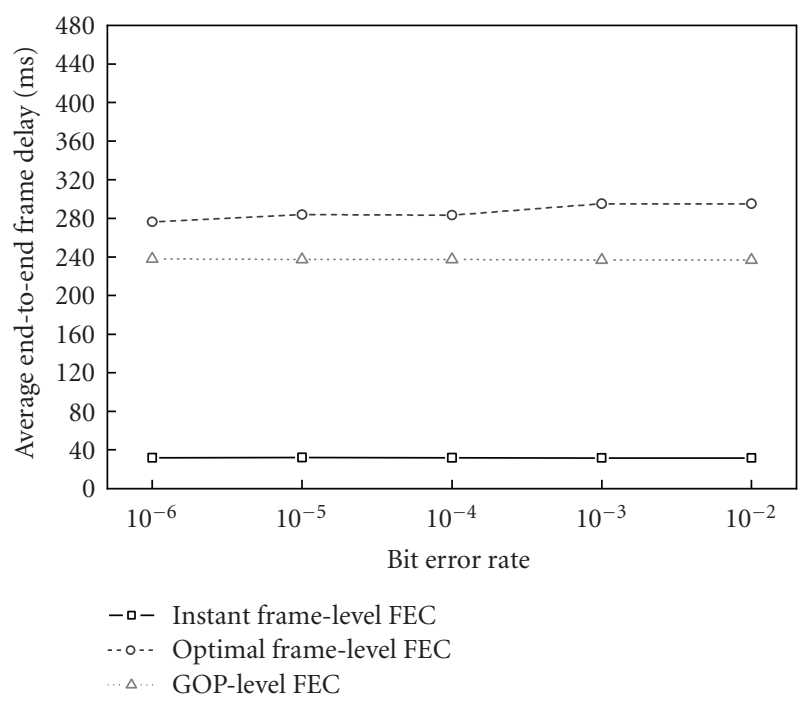

FIGURE 11: Comparison of end-to-end frame delays with varied bit error rate.

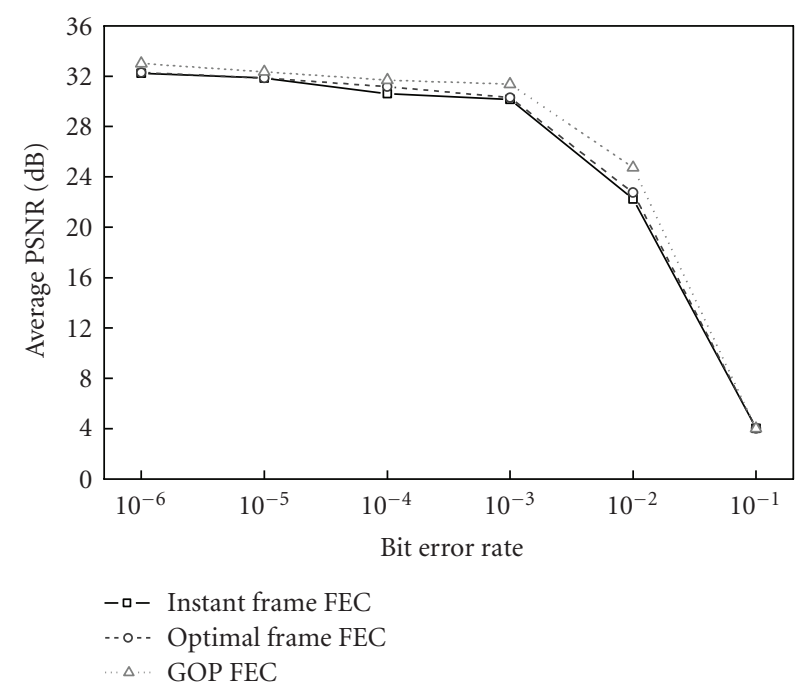

FIGURE 12: PSNR comparison with varied bit error rate.

gains transmission opportunities to cause a large end-toend frame delay. To conclude, the experimental results show that the instant frame-level scheme better preserves the timing aspects of real-time streaming video while achieving the near-highest video quality that the optimal frame-level scheme can obtain within the TCP-friendly rate constraints. Therefore, the proposed scheme transparently supports the robust video transmission on behalf of video applications and is suitable for the provision of high quality real-time video streaming with low delay.

\section{CONCLUSIONS}

This paper has developed a transparent QoS mechanism designated as TQM to transparently establish QoS sessions on behalf of multimedia applications over the wireless

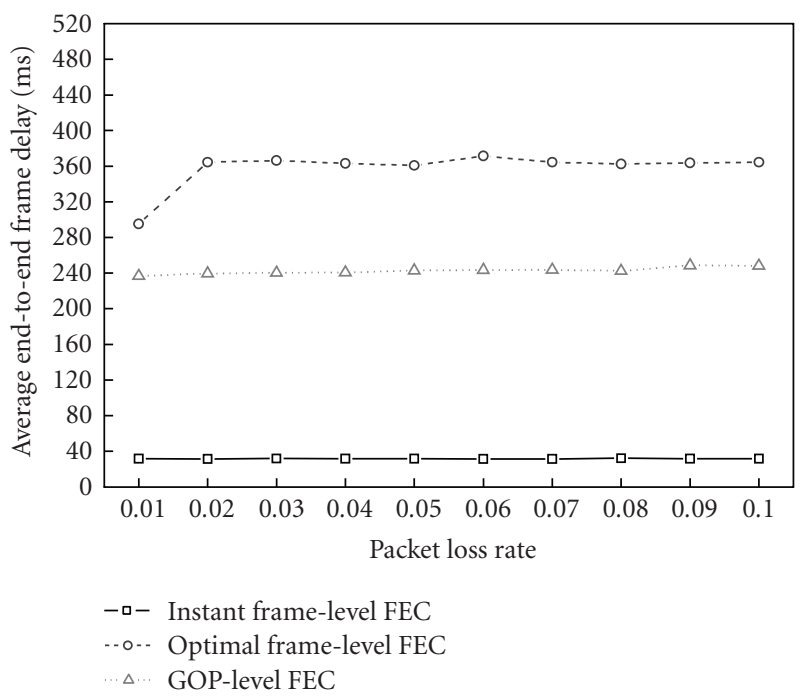

FIgure 13: Comparison of end-to-end frame delays with varied packet loss rate.

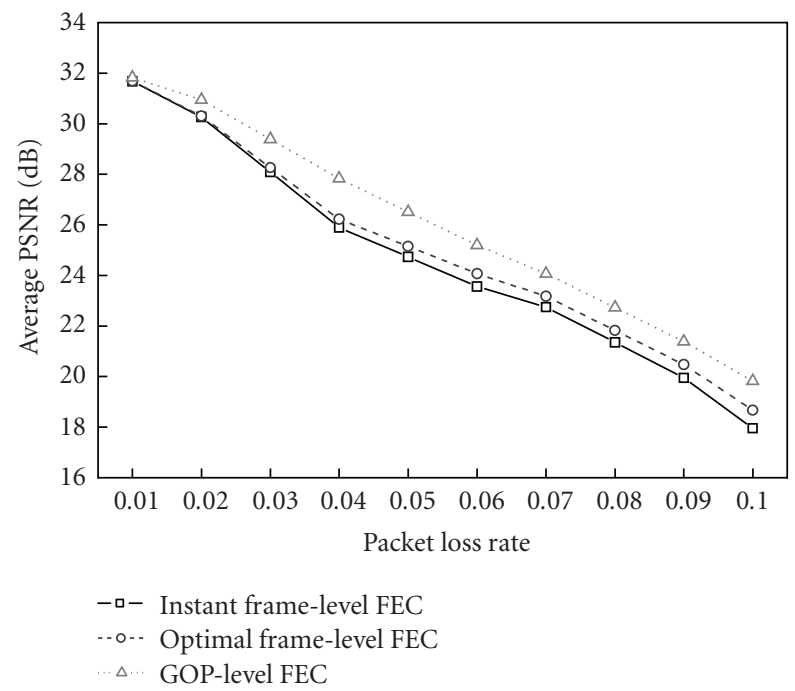

FIGURE 14: PSNR comparison with varied packet loss rate.

Internet. With no modification required to the existing legacy applications, TQM uses an efficient packet redirection scheme to divert user-specified flows for QoS enhancement processing and provides a suitable platform for the implementation of different QoS EMs. In TQM, EMs may function either independently or with other EMs to improve the overall QoS enhancement. As a first step, this study has discussed the implementation involved in integrating available EMs to support the transparent loss recovery based on FEC. The FEC-on-TQM module uses an instant framelevel FEC technique to achieve the near-highest video quality that the optimal frame-level scheme can obtain within the TCP-friendly rate constraints for real-time streaming video applications. The experimental results have shown that: (1) the overheads incurred by the proposed TQM scheme are minor compared to the total transmission and processing 
delays incurred along the path between the sender and the receiver, and (2) FEC-on-TQM successfully integrates several EMs to carry out the proposed instant frame-level FEC technique, resulting in a low-delay, good-visual-quality video experience. Future studies include the testing of networkadaptive video applications, such as Helix video server, on FEC-on-TQM, and the integration of EMs to further improve the overall QoS of diverse multimedia applications over the wireless Internet.

\section{REFERENCES}

[1] J. Wroclawski, The use of RSVP with IETF Integrated Services, RFC 2210, September 1997.

[2] S. Black, D. Black, M. Carlson, E. Davies, Z. Wang, and W. Weiss, "An architecture for differentiated services," RFC 2475, December 1998.

[3] Q. Zhang, W. Zhu, and Y.-Q. Zhang, "End-to-end QoS for video delivery over wireless Internet," Proceedings of the IEEE, vol. 93, no. 1, pp. 123-133, 2005.

[4] Quality of service (QoS) concept and architecture, 3GPP TS 23.107, September 2003.

[5] IEEE Standard, "Local and metropolitan area networksspecific requirements part 11: wireless LAN medium access control (MAC) and physical layer (PHY) specifications amendment 8: medium access control (MAC) quality of Service enhancements," pp.1-189, 2005.

[6] IEEE Standard, "802.16-2004 part 16: air interface for fixed broadband wireless access systems," October 2004.

[7] K. Nahrstedt, D. Xu, D. Wichadakul, and B. Li, "QoS-aware middleware for ubiquitous and heterogeneous environments," IEEE Communications Magazine, vol. 39, no. 11, pp. 140-148, 2001.

[8] Y.-B. Miao, W.-S. Hwang, and C.-K. Shieh, "A transparent deployment method of RSVP-aware applications on UNIX," Computer Networks, vol. 40, no. 1, pp. 45-56, 2002.

[9] L. Munoz, M. Garcia, J. Choque, R. Aguero, and P. Mahonen, "Optimizing Internet flows over IEEE 802.11b wireless local area networks: a performance-enhancing proxy based on forward error correction," IEEE Communications Magazine, vol. 39, no. 12, pp. 60-67, 2001.

[10] K. Park and W. Wang, "QoS-sensitive transport of real-time MPEG video using adaptive redundancy control," Computer Communications, vol. 24, no. 1, pp. 78-92, 2001.

[11] O. Ait-Hellal, E. Altman, A. Jean-Marie, and I. A. Kurkova, "On loss probabilities in presence of redundant packets and several traffic sources," Performance Evaluation, vol. 36-37, pp. 485-518, 1999.

[12] K. Park and W. Willinger, Self-Similar Network Traffic and Performance Evaluation, Wiley-Interscience, New York, NY, USA, 2000.

[13] S. Floyd and K. Fall, "Promoting the use of end-to-end congestion control in the Internet," IEEE/ACM Transactions on Networking, vol. 7, no. 4, pp. 458-472, 1999.

[14] H. Wu, M. Claypool, and R. Kinicki, "Adjusting forward error correction with temporal scaling for TCP-friendly streaming MPEG," ACM Transactions on Multimedia Computing, Communications, and Applications, vol. 1, no. 4, pp. 315-337, 2005.

[15] Y. Yuan, B. F. Cockburn, T. Sikora, and M. Mandal, "Efficient allocation of packet-level forward error correction in video streaming over the Internet," Journal of Electronic Imaging, vol. 16, no. 2, Article ID 023012, 12 pages, 2007.
[16] M. Baldi and Y. Ofek, "End-to-end delay analysis of videoconferencing over packet-switched networks," IEEE/ACM Transactions on Networking, vol. 8, no. 4, pp. 479-492, 2000.

[17] Linux IP firewall HOWTO,, http://www.linuxfaq.com /LDP/ HOWTO/Firewall-HOWTO.html.

[18] Divert sockets, http://tldp.org/HOWTO/Divert- Sockets-mini-HOWTO.html\#toc6.

[19] R. Rejaie, M. Handley, and D. Estrin, "Quality adaptation for congestion controlled video playback over the Internet," ACM SIGCOMM Computer Communication Review, vol. 29, no. 4, pp. 189-200, 1999.

[20] S. Floyd, M. Handley, J. Padhye, and J. Widmer, "Equationbased congestion control for unicast applications," in Proceedings of the ACM Annual Conference of the Special Interest Group on Data Communication (SIGCOMM '00), pp. 43-56, Stockholm, Sweden, August-September 2000.

[21] M. Handley, S. Floyd, J. Pahdye, and J. Widmer, "TCP friendly rate control (TFRC): protocol specification,” RFC3448, January 2003.

[22] D. Wu, Y. T. Hou, W. Zhu, et al., "On end-to-end architecture for transporting MPEG-4 video over the Internet," IEEE Transactions on Circuits and Systems for Video Technology, vol. 10, no. 6, pp. 923-941, 2000.

[23] Q. Zhang, W. Zhu, and Y.-Q. Zhang, "Resource allocation for multimedia streaming over the Internet," IEEE Transactions on Multimedia, vol. 3, no. 3, pp. 339-355, 2001.

[24] Z. Wang, S. Banerjee, and S. Jamin, "Media-friendliness of a slowly-responsive congestion control protocol," in Proceedings of the 14th International Workshop on Network and Operating System Support for Digital Audio and Video (NOSSDAV '04), pp. 82-87, Cork, Ireland, June 2004.

[25] V. Samata, R. Olivera, A. Dixit, P. Aghera, P. Zerfos, and S. Lu, "Impact of video transcoding parameters on dynamic video transcoding," in Proceedings of the 1st International Conference on Communication System Software and Middleware (COMSWARE '06), New Delhi, India, January 2006.

[26] V. Arya and T. Turletti, "Accurate and explicit differentiation of wireless and congestion losses," in Proceedings of the 23rd International Conference on Distributed Computing Systems (ICDCS '03), Providence, RI, USA, May 2003.

[27] S. Biaz and N. Vaidya, "Discriminating congestion losses from wireless losses using inter-arrival times at the receiver," in Proceedings of the IEEE Symposium on Application-Specific Systems and Software Engineer (ASSET '99), pp. 10-17, Richardson, Tex, USA, March 1999.

[28] Y. Tobe, Y. Tamura, A. Molano, S. Ghosh, and H. Tokuda, "Achieving moderate fairness for UDP flows by path-status classification," in Proceeding of the 25th Annual IEEE Conference on Local Computer Networks (LCN '00), pp. 252-261, Tampa, Fla, USA, November 2000.

[29] H. Schulzrinne, S. Casner, R. Frederick, and V. Jacobson, "RTP: a transport protocol for real-time applications," RFC 3550, July 2003.

[30] F. Borgonovo and A. Capone, "Efficiency of error-control schemes for real-time wireless applications on the Gilbert channel," IEEE Transactions on Vehicular Technology, vol. 54, no. 1, pp. 246-258, 2005.

[31] A. Li, "RTP payload format for generic forward error correction," Internet Draft draft-ietf-avt-ulp-18, IETF, work in progress, June 2006.

[32] J. Rosenberg and H. Schulzrinne, "An RTP payload format for generic forward error correction," RFC 2733, December 1999. 
[33] J. Padhye, V. Firoiu, D. F. Towsley, and J. F. Kurose, "Modeling TCP reno performance: a simple model and its empirical validation," IEEE/ACM Transactions on Networking, vol. 8, no. 2, pp. 133-145, 2000.

[34] S. Gringeri, R. Egorov, K. Shuaib, A. Lewis, and B. Basch, "Robust compression and transmission of MPEG-4 video," in Proceedings of the 7th ACM International Conference on Multimedia (MULTIMEDIA '99), pp. 113-120, Orlando, Fla, USA, October 1999.

[35] The VideoLAN server, http://www.videolan.org/vlc/streaming .html.

[36] S. Cen, P. C. Cosman, and G. M. Voelker, "End-to-end differentiation of congestion and wireless losses," IEEE/ACM Transactions on Networking, vol. 11, no. 5, pp. 703-717, 2003. 